\section{MS3-P5 Introducing DUI, a graphical interface for DIALS}

Luis Fuentes-Montero ${ }^{1}$, James Parkhurst ${ }^{1}$, Markus Gerstel ${ }^{1}$, Richard Gildea ${ }^{1}$, Graeme Winter ${ }^{1}$, Melanie Vollmar ${ }^{1}$, David Waterman $^{2}$, Gwyndaf Evans ${ }^{1}$

1. Diamond Light Source Ltd, Harwell Science and Innovation Campus, Didcot, OX11 0DE, UK

2. CCP4, Research Complex at Harwell, Rutherford Appleton Laboratory, Didcot OX11 OFA, UK

email: luis.fuentes-montero@diamond.ac.uk

Given the usefulness of DIALS[1] as an integration software package and its command line utility nature, there is an obvious need for a tool that runs DIALS commands in a user-friendly and interactive way. There is also a need for visualization tools to help users diagnose the integration process. The aim of the DIALS User Interface (DUI) is to provide these tools to run and control DIALS. It should also act as a tool in teaching DIALS and data processing in general.

The DUI's layout has some features in common with iMOSFLM[2] and also follows the same workflow principals. The DUI's flexibility enables both the running of complicated commands with many parameters and simple commands using default parameters. The program is conceived in such a way that the user may never need to use the keyboard. While the user is adjusting parameters and choosing options with the mouse, the DUI is writing in real time the command line that should be run accordingly. The program generates graphics to help the user inspect the results. These graphics remain available in HTML5 format for later reuse, either for analysis or for publication.

The DUI is particularly helpful when an automated pipeline tool like $\mathrm{Xia2}$ [3] fails or gives unexpected results. It allows the user to run DIALS but in a controlled way, with the user taking over decision making.

References:

[1] David G. Waterman, Graeme Winter, James M. Parkhurst, Luis Fuentes-Montero, Johan Hattne,Aaron Brewster, Nicholas K. Sauter, Gwyndaf Evans (2013) "The DIALS framework for integration software", CCP4 newsletter, n 49, pg 16-19

[2] T. Geoff G. Battye, Luke Kontogiannis, Owen Johnson, Harold R. Powell and Andrew G. W. Leslie (2011) "iMOSFLM: a new graphical interface for diffraction-image processing with MOSFLM", Acta Cryst D, Vol 67, Part 4 pg 271-281

[3] G. Winter "xia2: an expert system for macromolecular crystallography data reduction" (2010) J. Appl. Cryst, Vol 43, pg 186-190

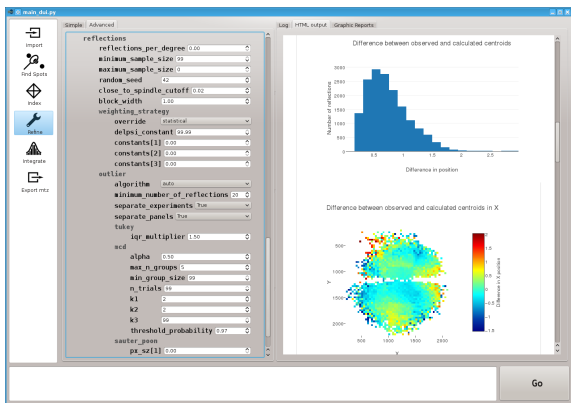

Figure 1. DUI showing diagnostic graphics after running refine job

Keywords: data integration, graphical user interface, data processing, software, DIALS, CCP4, macromolecular crystallography, collaboration 